\title{
Desenvolvimento de nova metodologia para aplicação de bioinseticidas no controle de borrachudos (Diptera: Simuliidae) em ribeirões com fluxo de água irregular
}

\author{
Development of new methodology for bioinseticide application for \\ black fly (Diptera: Simuliidae) control in rivers with irregular flow
}

\author{
Fernando Pereira dos Santos ${ }^{1}$; José Lopes ${ }^{2 *}$
}

\begin{abstract}
Resumo
Objetivando desenvolver adaptações metodológicas para aplicação de bioinseticida Teknar ${ }^{\circledR}$ no controle de simulídeos em ribeirões com fluxo de água irregular e identificar as espécies presentes, realizou-se experimentos nos ribeirões Cafezal, Cambé e Tamapuã, localizados no sul do Brasil. O primeiro, com fluxo de água regular, foi considerado como controle e os outros dois, com fluxos irregulares, foram as testemunhas para validação da nova metodologia proposta. Na metodologia tradicional, normalmente empregada para a aplicação do produto nos ribeirões considerados, foram alcançados as mortalidades larval de $99,13 \%, 59,76 \%$ e 13,87\%, respectivamente. Para a nova metodologia, considerando no cálculo da concentração do produto aplicado as irregularidades específicas as ribeirões Cambé e Tamapuã, essas percentagens aumentaram para 99,43\% e 99,32\%. Durante os experimentos foram coletadas 229.251 imaturos, sendo Grenieriella pruinosum e Chirostilbia pertinax, as mais abundantes nesta região.

Palavras-chave: Bacillus thuringiensis israelensis, controle biológico, Simuliidae
\end{abstract}

\begin{abstract}
The goals of this study were to develop methodological changes for applying the bio-pesticide Teknar (B) to control the black fly in streams with irregular flow and identify black fly species in three streams (Cafezal, Cambé, Tamapuã) in Southern Brazil. The only regular-flow stream Cafezal was the control stream; the other two streams had irregular flow and were selected to validate the new methodology. The traditional method for bio-insecticide application in the streams under study presented larval mortalities of $99.13 \%$ (Cafezal), 59.76\% (Cambé), and $13.87 \%$ (Tamapuã) . As a result of the new methodology, bio-insecticide concentration was determined according to the specific irregularities of the Tamapuã and Cambé streams and mortality percentages increased to $99.43 \%$ and $99.32 \%$, respectively. Among the 229,251 immature Simuliidae collected during the experiment, Grenieriella pruinosum and Chirostilbia pertinax were the most abundant.
\end{abstract}

Key words: Bacillus thuringiensis israelensis, biological control, Simuliidae

\footnotetext{
${ }^{1}$ Aluno do Programa de pós-Graduação em Agronomia na Universidade Estadual de Londrina, UEL. E-mail: nando@uel.br

2 Docente. do Departamento de Biologia Animal e Vegetal, Universidade Estadual de Londrina, UEL. Caixa Postal 6001, E-mail: jea@uel.br

* Autor para correspondência
} 


\section{Introdução}

Espécies de simulídeos estão envolvidas na transmissão de oncocercose humana principalmente na região norte do Brasil (LACEY; UNDER, 1984; CAVADOS et al., 2001; PETRY; LOZOVEI; EHLERS, 2006; NASCIMENTO et al., 2007). Nesta mesma região, existe uma outra moléstia conhecida como Pênfigo foliáceo ou "Fogo selvagem", doença específica do sistema imunológico. Indivíduos expostos aos insetos hematófagos, em especial ao Simulium nigrimanum Macquart 1838, apresentaram maior susceptibilidade a essa enfermidade (AOKI et al., 2005). No sudeste do Brasil, a principal importância volta-se para as questões sócioeconômicas, podendo afetar drasticamente o turismo e a qualidade de vida da população local (CAVADOS et al., 2001) e também a produção animal, causando perda de peso, a diminuição na produção de leite, podendo causar a morte de aves (CUNHA 2001).

Para o controle desse vetor, o método mais adequado é o biológico, utilizando-se bactérias do gênero Bacillus. Bacillus thuringiensis israelensis Berliner (B.t.i.) tem sido utilizada como princípio ativo de bioinseticida por sua patogenicidade e especificidade sobre larvas de borrachudos (LACEY; LACEY, 1981; BECKER et al., 1992; BROWN et al., 1998; 1999; 2000; RODRIGUES; WANDERLI; JOSÉ, 1998; NAYAR et al.,1999; CHUNG et al., 2001; FILLINGER; KNOLS; BECKER, 2003; POLLOM, 2003; CAVADOS et al., 2005).

Cada ribeirão tem sua característica geomorfológica, mas os modelos para cálculo de concentração do produto a ser aplicado são construídos baseados em águas correntes de forma uniforme podendo não serem adequados para todas as situações topográficas que acompanham os ribeirões. A estratégia de aplicação corresponde ao principal fator que garante o sucesso do programa, já que o B.t.i. está comprovado como bioinseticida eficiente.

Este trabalho teve como objetivo elaborar e testar uma variável metodológica para aplicação de bioinseticida a base de $B$. thuringiensis israelensis no controle de simulídeos, em ribeirões com características geomorfológicas acentuadas.

\section{Material e Métodos}

Os experimentos foram realizados em três ribeirões, Cafezal, Cambé e Tamapuã. A área estudada do ribeirão Cafezal está localizada no município de Rolândia-PR (23²2’30”S; $\left.51^{\circ} 23^{\prime} 30^{\prime \prime} \mathrm{W}\right)$, com mata ciliar reduzida e em algumas regiões com gramíneas atingindo suas margens. Esse ribeirão, tem sua nascente próxima a área urbana e, neste trecho, recebe forte influência gerada pela urbanização e ação antrópica, que já está minimizada na área estudada. Apresenta leito com poucas irregularidades que permite suas águas fluírem de forma contínua. Este ribeirão foi definido como controle para comparação da metodologia tradicional com a metodologia proposta.

O ribeirão Cambé está localizado no município de Londrina, PR, Brasil (2317'15'S; 51 13' 58'W). A bacia deste ribeirão encontra-se quase toda urbanizada, com mata ciliar reduzida ou ausente. De modo geral, este córrego é influenciado por material particulado proveniente da auto estrada e de esgotos clandestinos industriais e domésticos. Apresenta leito irregular com trechos de águas lóticas, corredeiras e pequenas cachoeiras que formam, após a queda, piscinas naturais.

O ribeirão Tamapuã está localizado no município de Arapongas-Pr (23⒉'45”S; 51'34'25'W), em terreno íngreme, resultando em uma topografia com a presença de piscinas naturais de dimensões consideráveis. Por estar localizado no perímetro urbano, esse ribeirão apresenta mata ciliar reduzida e presença de entulhos e lixo doméstico ao longo de seu leito.

Foram realizados cálculos de vazão dos córregos, tomando-se como base uma extensão de $10 \mathrm{~m}$. Neste trecho, a largura foi determinada de metro a metro, perfazendo dez medidas. Em cada uma destas seções, a profundidade foi medida a cada $20 \mathrm{~cm}$ a partir da margem. Este procedimento forneceu em torno de 50 medidas de profundidade para o trecho. A velocidade da água foi calculada utilizando-se flutuadores (bola de tênis de mesa), previamente preenchido com $25 \mathrm{~mL}$ de água. Este flutuador 
foi solto no marco zero e o tempo para alcançar o ponto final $(10 \mathrm{~m})$ foi determinado utilizando um cronômetro da marca Technos ${ }^{\circledR}$ - Cronus. O número de repetições dependeu da largura do ribeirão, já que, o flutuador foi solto de $20 \mathrm{em} 20 \mathrm{~cm}$ em toda a largura do ponto zero. De posse destas medidas, calculou-se a vazão de acordo com a equação I:

Equação I:

$$
\mathrm{Q}=\frac{\operatorname{Lm} \times \operatorname{Pm} \times C \times 0,85}{\operatorname{Tm}}
$$

Onde: $\mathrm{Q}=$ vazão $\left(\mathrm{m}^{3} \cdot \mathrm{min}^{-1}.\right) ; \mathrm{C}=$ comprimento (m); Lm = largura média (m); Pm = profundidade média (m); Tm = tempo médio (m); 0,85 é uma constante que serve para corrigir o atrito da água com o fundo.

Calculada a vazão, utilizou-se a tabela produzida pela Divisão de Zoonoses da Secretaria de Saúde e Meio Ambiente do estado do Rio Grande do Sul (Tabela 1) (RIO GRANDE DO SUL, 2006) para determinar a concentração do produto a ser aplicado e seu carreamento. Para o cálculo da quantidade de produto a ser aplicado, foi multiplicado o valor da vazão pela concentração correspondente na tabela.
O ribeirão Cafezal apresenta uma característica topográfica típica de criadouros naturais de borrachudos, ou seja, pouca profundidade, irregularidades topográficas, e substratos adequados para oviposição. Baseando-se nestas características, o experimento, que realizou-se no período de maio a junho de 2006, foi direcionado a utilizá-lo como um ribeirão comparativo aos experimentos descritos anteriormente. Foram realizadas medidas de vazão, obtendo-se um carreamento de $250 \mathrm{~m}$ (Figura 1). O ponto testemunha para coleta de larvas foi instalado logo no início do trecho e, a dois metros abaixo deste, o ponto de aplicação do bioinseticida. O segundo ponto de coleta de larvas (ponto I) foi estabelecido a dois metros abaixo do ponto de aplicação, e 150m após o ponto I, fixou-se o ponto II. No final do carreamento (250m após a testemunha) foi instalado o ponto III, utilizado para verificar a eficiência do cálculo de vazão. Instalou-se também um quarto ponto, $100 \mathrm{~m}$ abaixo do ponto III, para verificação de um possível aumento no carreamento do produto. $\mathrm{O}$ volume de biolarvicida aplicado foi de $150 \mathrm{ml}$, valor obtido pela tabela sugerida pela Divisão de Zoonoses e Vetores da Secretaria da Saúde e Meio Ambiente do Rio Grande do Sul (RIO GRANDE DO SUL, 2006).

Tabela 1. Cálculo de dosagem de aplicação e carreamento de produtos biológicos, através dos valores de vazão.

\begin{tabular}{ccc}
\hline Vazão $\left(\mathbf{m}^{3} / \mathbf{m i n}\right)$ & Concentração $(\mathbf{p p m})$ & Carreamento $(\mathbf{m})$ \\
\hline 0,01 a 0,31 & 50 & $50(01)$ \\
0,32 a 0,62 & 50 & $60(01)$ \\
0,63 a 1,25 & 45 & $75(01)$ \\
1,26 a 2,50 & 40 & $125(02)$ \\
2,51 a 5,00 & 30 & $250(02)$ \\
5,01 a 10,0 & 20 & $500(02)$ \\
10,01 a 15,00 & 15 & $750(02)(03)$ \\
15,01 a 20,00 & 12 & $1000(01)(03)$ \\
\hline
\end{tabular}

(01) Limites máximos para não-agressão aos organismos não alvos, recomenda-se aplicar o produto somente em situações extremas, onde ocorra um elevado nível de infestação larval e ataque intenso de insetos ao homem. Ao aplicá-lo deve-se evitar cascatas e empoçamentos.

(02) Intervalos de aplicações normais de rotina.

(03) Intervalos onde se deve empregar dois (2) regadores para as aplicações

* Se o cálculo da dosagem for inferior a $5 \mathrm{~mL}$, aplica-se $5 \mathrm{~mL}$ como dose mínima.

Fonte: Divisão de Zoonoses e Vetores da Secretaria da Saúde e Meio Ambiente do Rio Grande do Sul. Simulídeos: Programa Estadual Rio Grande do Sul - Brasil, 2006. 


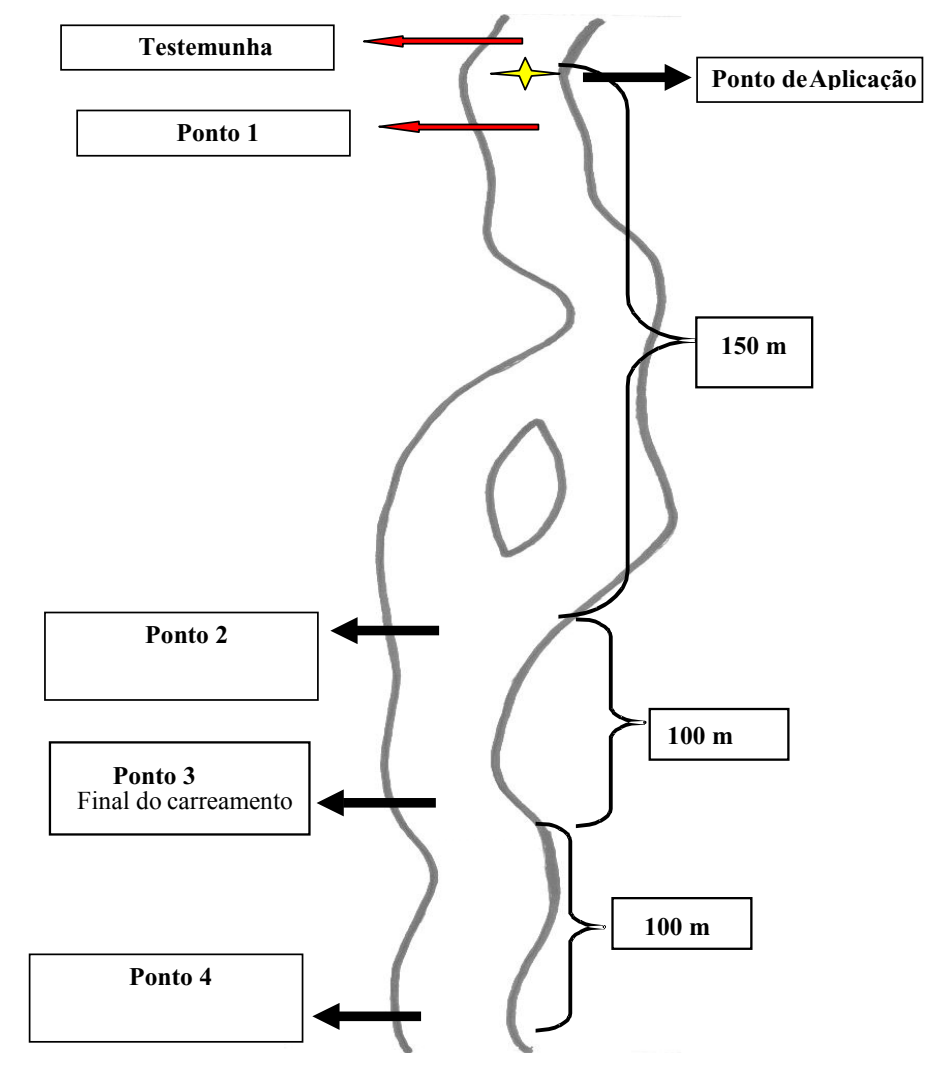

Figura 1. Geomorfologia do ribeirão Cafezal, indicando os pontos de aplicação do B. thuringiensis israelensis e os pontos de coleta de imaturos de Simuliidae, na metodologia tradicional

Em todos os pontos, inclusive no ponto controle, instalou-se uma corda de nylon no sentido transversal de margem a margem, e nesta foram amarradas seis fitas (fitilhos) de polietilieno e polipropileno de cor verdes, com $2,1 \mathrm{~cm}$ de largura e $100 \mathrm{~cm}$ de comprimento, que serviram como local de fixação para as formas imaturas de simulídeos. As fitas, após instaladas ficaram 15 dias expostas para colonização por borrachudos.

Para as coletas das amostras foram retiradas três fitas alternadas momentos antes da aplicação do biolarvicida, em cada situação, e as três fitas restantes no dia seguinte, 24 horas após a aplicação do produto.

As aplicações foram feitas quinzenalmente, utilizando-se um regador, no qual foi colocado o produto na quantidade determinada pelos cálculos acima e em seguida completado com água até atingir cinco litros, volume estabelecido pela metodologia tradicional. Esta solução foi agitada e depois aplicada na superfície da água por toda a largura do ponto estipulado. O tempo usado para aplicação foi de um minuto a um minuto e meio. Tempo descrito por Lacey e Undeen (1984) como o mais eficiente.

O produto utilizado para controle em todos os três ribeirões foi o biolarvicida Teknar ${ }^{\circledR} 3.000 \mathrm{AAU} /$ $\mathrm{mg}$, lote 13700-3381 - 04-4849/R2, com número de registro no Ministério da Saúde 3.0119.0035, na formulação de suspensão aquosa concentrada em $1,6 \%$, onde seu princípio ativo é a bactéria Bacillus thuringiensis subsp. israelensis.

No ribeirão Cambé, para cada trecho a ser aplicado, foram instalados três pontos de coleta de imaturos de borrachudos; ponto controle localizado $12 \mathrm{~m}$ acima do ponto inicial de aplicação; ponto I, dois metros a baixo da liberação do produto e ponto II no final do valor do carreamento. 
Neste ribeirão o experimento foi dividido em duas etapas. Em um primeiro momento, no período de janeiro de 2006 a março de 2006, a aplicação do produto foi realizada seguindo os valores de carreamento indicados na Tabela 1 , ou seja, a aplicação, foi realizada independente das irregularidades do ribeirão (Figura 2A). Neste foram aplicados $239 \mathrm{~mL}$ no ponto 1 e $254 \mathrm{~mL}$ no ponto 2 , trecho 1 e trecho 2, respectivamente.

$\mathrm{Na}$ segunda fase do experimento (abril e maio de 2006) foi estabelecida e testada modificações na metodologia para aplicação, onde foram levadas em consideração as quedas d'águas com a formação de piscinas naturais, o que diminui significativamente a velocidade da água e a profundidade do leito do curso d'água (Figura 2B). Esta modificação da metodologia, consiste em divisão da dose obtida pela metodologia tradicional, pela distância de carreamento do produto. De acordo com o resultado obtido, multiplica-se por uma constante.

Por esta nova proposta ficou estabelecido três pontos de aplicação e os valores de dose foram os seguintes: Ponto 1: $57,4 \mathrm{~mL}$ de produto para um carreamento de 40m até o remanso; Ponto 2: $161 \mathrm{~mL}$ para um carreamento de $338 \mathrm{~m}$; Ponto $3: 75 \mathrm{~mL}$ para um carreamento de $52 \mathrm{~m}$.

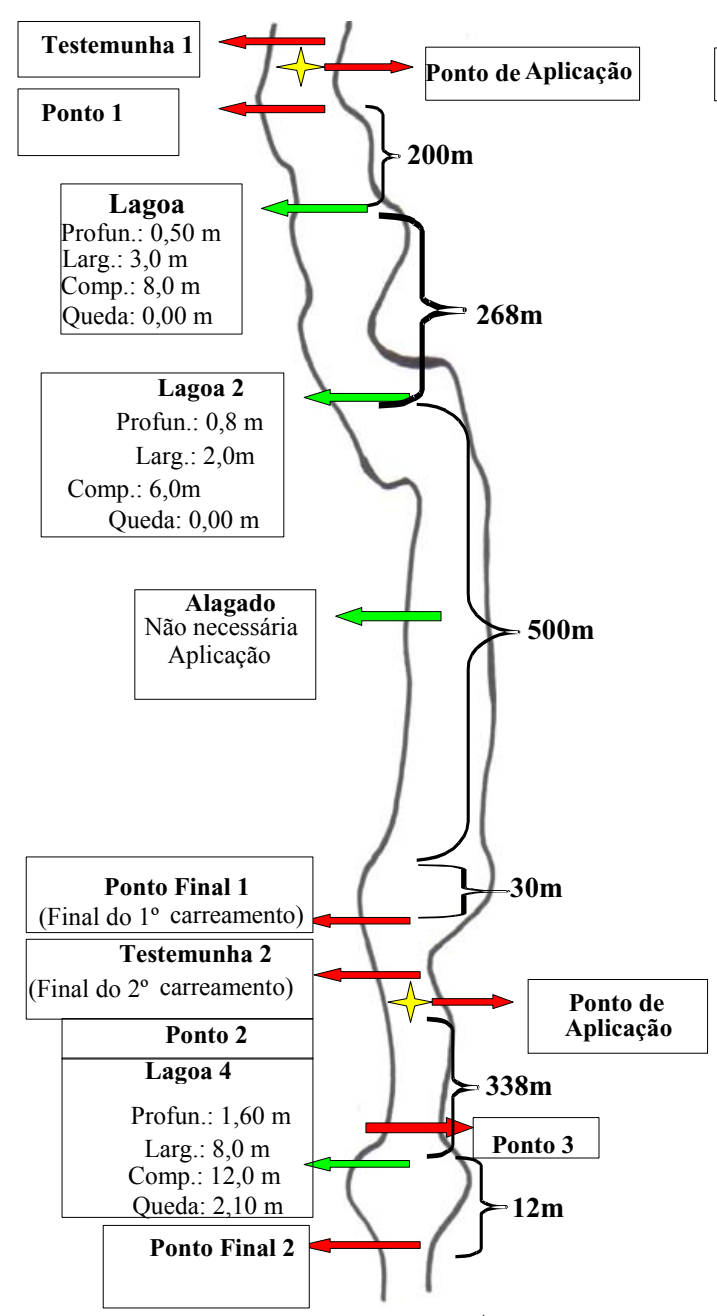

A

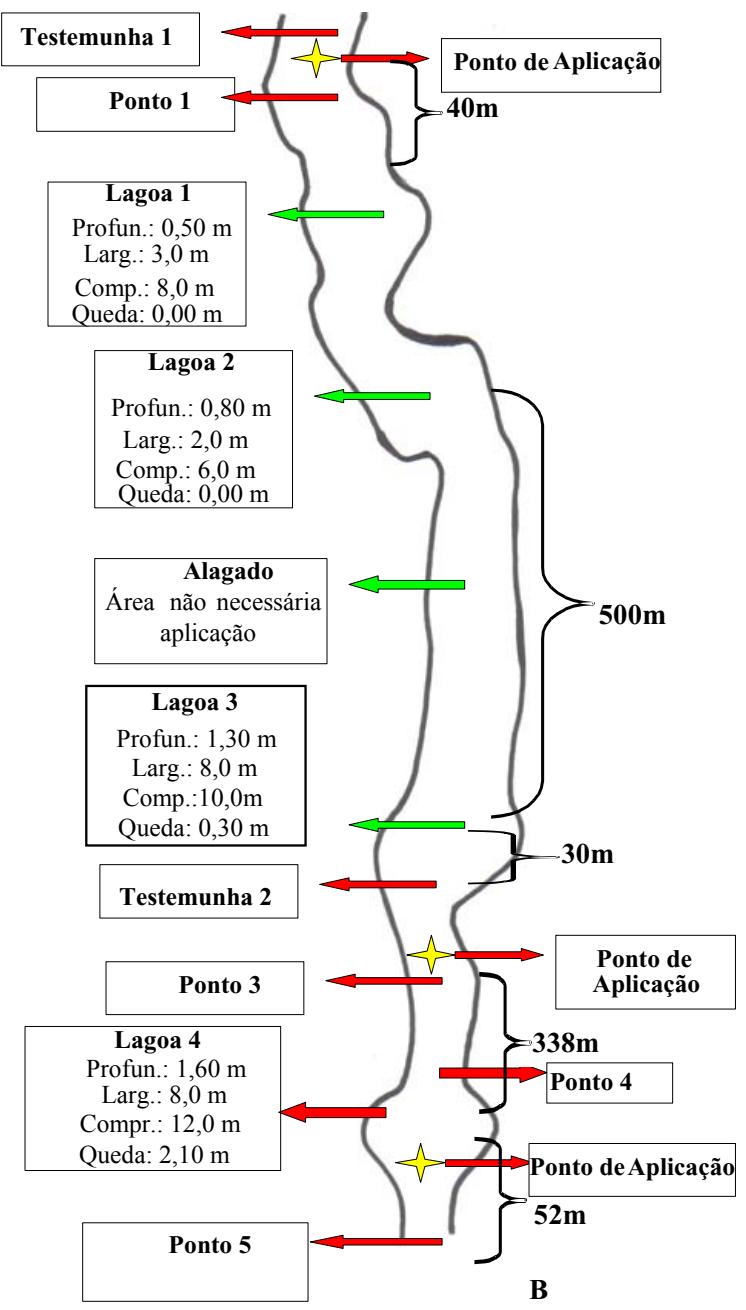

Figura 2. Geomorfologia do ribeirão Cambé, indicando as variações no seu leito, os pontos de aplicação do $B$. thuringiensis israelensis e os pontos de coleta de imaturos de Simuliidae. A) Metodologia tradicional; B) Nova Metodologia 


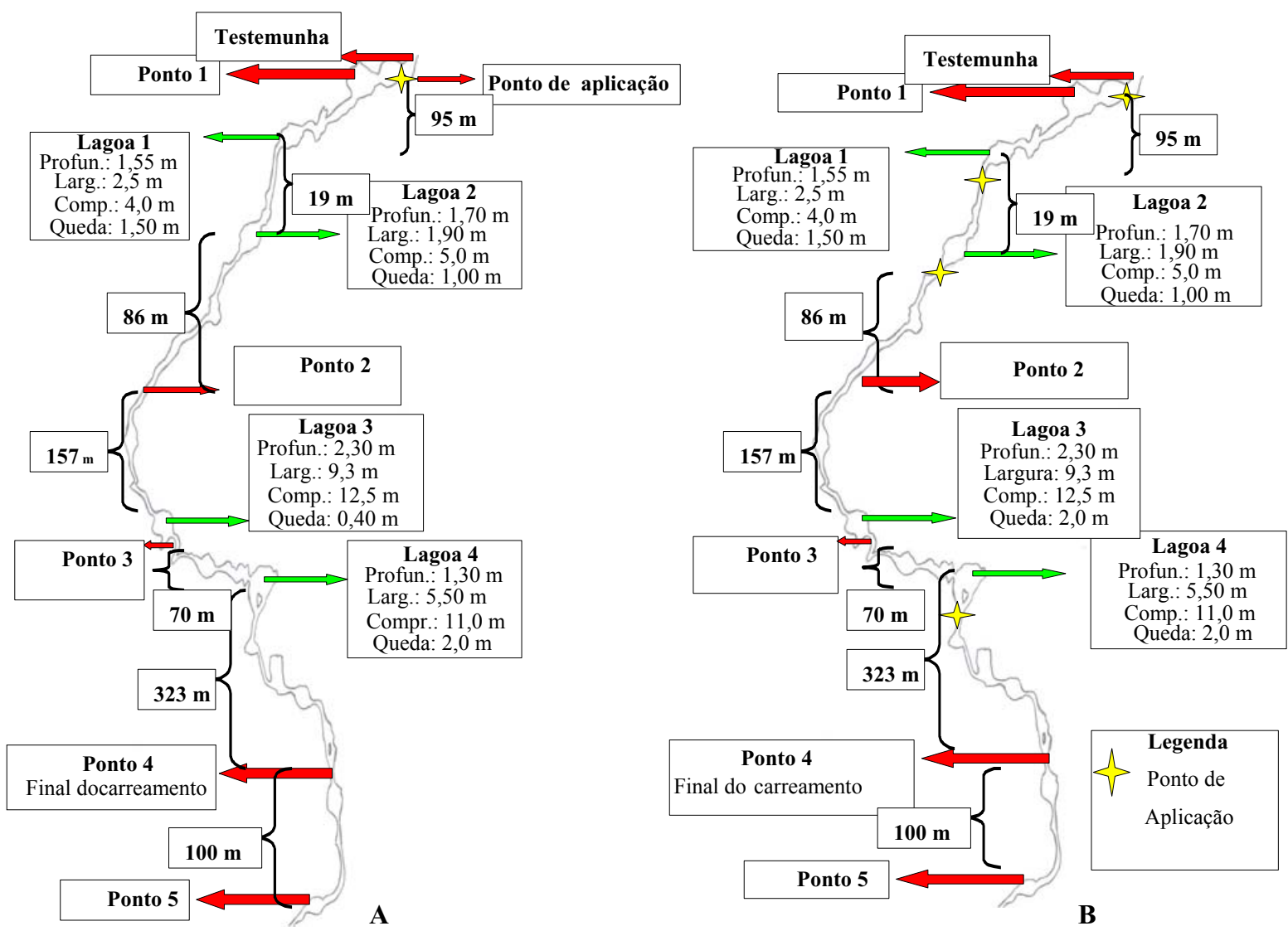

Figura 3. Geomorfologia do ribeirão Tamapuã, indicando as variações no seu leito, os pontos de aplicação do $B$. thuringiensis israelensis, e os pontos de coleta de imaturos de Simuliidae. A) Metodologia tradicional; B) Nova Metodologia.

O experimento no ribeirão Tamapuã foi semelhante ao do alto Ribeirão Cambé, ou seja, foi dividido em duas etapas: em um primeiro momento que compreendeu de 13 de outubro de 2006 a 04 de novembro do mesmo ano, e em um segundo momento que foi de 10 a 25 de novembro de 2006. Este ribeirão foi utilizado como uma repetição do experimento realizado no ribeirão Cambé para validação da nova proposta de metodologia.

Para o primeiro momento foram feitas as medidas de vazão, obtendo-se um valor de $750 \mathrm{~m}$ de carreamento para $170 \mathrm{~mL}$ de produto a ser aplicado (Figura 3A). Instalou-se 6 pontos de análise ao longo do ribeirão. Após $10 \mathrm{~m}$ da testemunha instalou-se o ponto I de coleta de dados, o ponto II foi localizado a $250 \mathrm{~m}$ do ponto zero, o ponto III a $500 \mathrm{~m}$, o ponto
IV a $600 \mathrm{~m}$, o ponto $\mathrm{V}$ a $750 \mathrm{~m}$ e o ponto VI a $850 \mathrm{~m}$. Este último ponto foi instalado com o objetivo de verificar o possível aumento no carreamento do produto.

Desta forma seguindo os cálculos da nova proposta de metodologia o trecho do ribeirão Tamapuã foi subdividido em cinco pontos de aplicação com as seguintes doses: ponto I: $131 \mathrm{~mL}$; ponto II: $26 \mathrm{~mL}$; ponto III: $112 \mathrm{~mL}$; ponto IV: $83 \mathrm{~mL}$ e ponto V: $92 \mathrm{~mL}$, para carreamento de $95 \mathrm{~m}, 19 \mathrm{~m}$, $86 \mathrm{~m}, 227 \mathrm{~m}$ e $482 \mathrm{~m}$, respectivamente.(Figura 3B).

As amostras de larvas e pupas coletadas em todos os experimentos foram contadas com o auxílio de uma lupa Olympus ${ }^{\circledR}$, e posteriormente armazenadas. Em cada procedimento de coleta cerca de $10 \%$ (dez) das larvas e pupas eram montadas em 
lâminas para identificação específica dos imaturos. A identificação foi baseada em características morfológicas específicas das manchas de apótemas cefálicos e em ramificações dos filamentos branquiais contidos em histoblastos. As chaves dicotômicas de Coscarón e Wygodzinsky (1984), Coscarón (1991), Strieder, Corseuil e Py-Danyel (1992) e Hamada, Ale-Rocha e Luz 2003, serviram de suporte técnico para esta identificação. Uma amostra das larvas foram enviadas para especialista para confirmação das espécies.

As espécies identificadas foram nomeadas de acordo com Strieder (2004), onde sugere a elevação de subgênero para o nível de gênero para as espécies de Simuliidae Newman, 1834.

Os dados obtidos foram analisados estatísticamente pelo o teste de Tukey no nível $5 \%$ de significância como forma de comparar as duas metodologias de controle. A diversidade e abundância das espécies existentes também foram apresentadas.

\section{Resultados e Discussão}

Foram coletados 229.251 imaturos de Simuliidae, dos quais $189.950(82,86 \%)$ de larvas e 39.301 $(17,14 \%)$ de pupas. Foram identificados cerca de 10\% (22.925 imaturos, entre estágio de pupa e larvas de último estádio) do total coletado. Ao se estudar a ação do bioinseticida a ser utilizado no controle de insetos, faz-se necessário conhecer as espécies alvo.

As espécies coletadas no ribeirão Cafezal foram Chirostilbia pertinax Kollar, 1832 com 81,72\%, Inaequalium inaequale Paterson e Shannon, 1927 com 8,22\%, Chirostilbia subpallida Lutz, 1910 com 7,54\%, Psaroniocompsa incrustata Lutz,1910 com 1,73\% e Grenieriella pruinosum Lutz, 1910 com 0,76\%. No ribeirão Cambé obteve-se as seguintes espécies: G. pruinosum representando 70,73\%,C. subpallida com 20,78\%, C. pertinax com 7,18\%, I. inaequale com $0,84 \%$, P. incrustata com $0,36 \%$ e
Hemicnetha rubrithorax Lutz, 1909 com 0,09\%. Já no ribeirão Tamapuã as espécies foram: $C$. pertinax com 78,36\%, G. pruinosum com 13,28\%,C. subpallida com 5,45\%, P. incrustata com 1,29\%, H. rubrithorax com $0,81 \%$ e I. inaequale com $0,78 \%$. G. pruinosum foi a espécie predominante no ribeirão Cambé. Segundo Coscarón (1991), referindo-se a esta espécie, afirma que é comumente encontrada em cursos d'água com pouca profundidade, águas cristalinas e fortes correntezas. Neste experimento a espécie foi a mais coletada no ribeirão Cambé que está dentro da área urbana e recebe descarga de esgoto industrial e doméstico. C. pertinax parece ser uma das espécies mais favorecidas com o aumento da poluição dos ribeirões em áreas rurais. Um dos problemas ambientais que favorecem o desequilíbrio populacional das espécies é o elevado despejo de matéria orgânica nos cursos d'água, principalmente devido ao tratamento inadequado dos dejetos oriundos da criação de animais domésticos (STRIEDER; SANTOS JUNIOR; PES, 2002; STRIEDER, 2005). Esta adaptabilidade pode justificar ser esta a espécie dominante nos ribeirões Cafezal e Tamapuã que recebem resíduos orgânicos oriundos da área urbana. Esta espécie é fortemente antropofílica e é incriminada como a de maior importância médica sanitária na região sul do país (STRIEDER; SANTOS; VIEIRA, 2006).

As espécies encontradas nesta região de estudo são semelhantes as coletadas no estado do Rio Grande do Sul (STRIEDER; SANTOS; VIEIRA, 2006) e de Curitiba - Paraná (PETRY et al., 2004; LOZOVEI; PETRY; SANTOS NETO, 2004).

No ribeirão Cafezal, considerado mais uniforme, obteve-se mortalidade de 99,13\% quando aplicado $150 \mathrm{mLdeproduto}$ Teknar ${ }^{\circledR}$ comanálise 24 horas após a aplicação, para um carreamento de $250 \mathrm{~m}$ (Tabela 2). Pode-se observar que o carreamento estabelecido pela metodologia tradicional foi eficiente, havendo mortalidade por todo o trecho estabelecido pela fórmula de cálculo de carreamento, mas não controlou no ponto estabelecido além da distância 
calculada (que consistia em um ponto localizado $100 \mathrm{~m}$ abaixo do carreamento determinado). Para as condições de fluxo contínuo de água do ribeirão, como no Cafezal, o resultado de controle foi ideal, podendo ser utilizada a metodologia tradicional com sucesso (Tabela 2).

Tabela 2. Controle de imaturos de Simuliidae no ribeirão Cafezal, Rolândia-Pr, pelo produto Teknar ${ }^{\circledR}$, pela metodologia tradicionalmente utilizada para cálculo de dosagem

\begin{tabular}{|c|c|c|c|c|c|c|c|c|c|c|c|c|}
\hline \multirow{3}{*}{ Pontos de coleta } & \multicolumn{12}{|c|}{ Datas de Coleta } \\
\hline & \multicolumn{4}{|c|}{ 15/Mai - 0 hr 16/Mai - 24 hr } & \multicolumn{2}{|c|}{ 29/Mai - 0 hr } & \multicolumn{2}{|c|}{ 30/Mai - 24 hr } & \multicolumn{2}{|c|}{ 12/Jun - 0 hr } & \multicolumn{2}{|c|}{ 13/Jun - 24 hr } \\
\hline & Larva & Pupa & Larva & Pupa & Larva & Pupa & Larva & Pupa & Larva & Pupa & Larva & Pupa \\
\hline Testemunha1 & 1184 & 30 & 1098 & 33 & 984 & 0 & 979 & 0 & 514 & 119 & 503 & 131 \\
\hline Ponto 1 & 2670 & 0 & 3 & 0 & 1804 & 1 & 13 & 0 & 878 & 51 & 9 & 33 \\
\hline Ponto 2 & 1198 & 3 & 15 & 1 & 838 & 0 & 19 & 2 & 522 & 16 & 8 & 9 \\
\hline Ponto 3 & 1231 & 5 & 12 & 0 & 1108 & 0 & 17 & 3 & 977 & 4 & 2 & 20 \\
\hline Ponto 4 & 1077 & 0 & 894 & 0 & 871 & 19 & 766 & 0 & 411 & 39 & 377 & 55 \\
\hline TOTAL & 5099 & 8 & 30 & 1 & 3750 & 1 & 49 & 5 & 2377 & 71 & 19 & 62 \\
\hline
\end{tabular}

Observação: o ponto 4 está localizado $100 \mathrm{~m}$ além do carreamento. O total não inclui os pontos: testemunha e ponto 4 .

Já no ribeirão Cambé, na utilização da metodologia tradicional, o ribeirão foi dividido em dois trechos (Figura 2A), com carreamento calculado para $1000 \mathrm{~m}$. Utilizou-se de $239 \mathrm{~mL}$ e $254 \mathrm{~mL}$ nos trecho 1 e trecho 2 , respectivamente, já que a vazão foi diferente para os dois trechos. Obteve-se 59,76\% de diminuição da quantidade de larvas de simulídeos após 24 horas da aplicação (Tabela 3).

$\mathrm{Na}$ segunda fase do experimento (somente para os ribeirões Cambé e Tamapuã), o método de aplicação de bioinseticida seguiu a nova proposta de metodologia, já que o leito dos ribeirões apresentavam-se irregulares.

A nova proposta de metodologia, consistiu em calcular os valores de vazão e carreamento da mesma forma como para o método anterior. Obtendo-se o valor do carreamento e consequentemente o valor da dose a ser aplicada. Em seguida subdividiu-se esta dose pela distância em metros dos trechos entre duas piscinas naturais, utilizando-se a equação II:
Equação II:

$$
\mathrm{D} / \mathrm{M}=\frac{\text { Dose do produto }}{\text { Carreamento }(\mathrm{m})}
$$

$\mathrm{D} / \mathrm{M}=$ Dose de produto por metro de ribeirão(mL/m)

A dose do produto e o carreamento são valores encontrados segundo a metodologia padrão

Uma vez obtido a quantidade do produto para aquele trecho delimitado, multiplica-se por uma constante seis quando a distância do ponto de aplicação à irregularidade do leito do córrego (piscina natural) for inferior ou igual a $200 \mathrm{~m}$. Quando a distância for superior a esta multiplica-se pela constante 2. Estas constantes foram obtidas através de testes preliminares para a obtenção de uma dose letal suficiente para matar $95 \%$ da população larval, partindo-se do valor de dose encontrado na relação $\mathrm{D} / \mathrm{M}$, visto anteriormente. 
Equação III:

$$
\mathrm{D} / \mathrm{M}=\frac{\text { Dose do produto }}{\text { Carreamento }(\mathrm{m})} \mathrm{x}
$$

$\mathrm{R}=$ constante $(6$ para distâncias de carreamento menores que $200 \mathrm{~m}$ e 2 quando a distância for superior a 200m)

A aplicação foi realizada da seguinte forma: marcou-se o ponto inicial do córrego (ponto de aplicação) e a distância deste até a primeira irregularidade (remanso - dentro do carreamento). O valor desta distância (X) foi multiplicado pelo $\mathrm{D} / \mathrm{M}$, obtendo-se a dose a ser aplicada neste trecho, seguindo-se este procedimento até o final do carreamento. Quando o valor do carreamento alterar, realiza-se novo cálculo de dose seguindo o mesmo procedimento.

Nesta fase, o ribeirão Cambé, formado por áreas alagadas com muita vegetação e pequenas quedas de água com formação de piscinas naturais de águas lênticas. A dose para o trecho 1 foi calculada desconsiderando a área alagada $(57,6 \mathrm{~mL})$, e no trecho 2 foi dividida em 2 pontos de aplicação (Figura 2B), sendo aplicado 165,0 mL e 75,0 mL, nos ponto 2 e ponto 3 , respectivamente. Nesta, foi obtida um controle de $99,43 \%$ quando analisada 24 horas após a aplicação do produto (Tabela 4).

Tabela 3. Controle de imaturos de Simuliidae no ribeirão Cambé, Londrina-PR, pelo produto Teknar ${ }^{\circledR}$, pela metodologia tradicionalmente utilizada para cálculo de dosagem

\begin{tabular}{|c|c|c|c|c|c|c|c|c|c|c|c|c|}
\hline \multirow{3}{*}{$\begin{array}{c}\text { Pontos de } \\
\text { Coleta }\end{array}$} & \multicolumn{12}{|c|}{ Datas de coleta } \\
\hline & \multicolumn{2}{|c|}{ 10/Jan - 0 hr } & \multicolumn{2}{|c|}{ 11/Jan - 24 hr } & \multicolumn{2}{|c|}{ 24/Jan - 0 hr } & \multicolumn{2}{|c|}{ 25/Jan - 24 hr } & \multicolumn{2}{|c|}{ 06/Mar - 0 h } & \multicolumn{2}{|c|}{ 07/Mar - 24 hr } \\
\hline & Larva & Pupa & Larva & Pupa & Larva & Pupa & Larva & Pupa & Larva & Pupa & Larva & Pupa \\
\hline Testemunha1 & 603 & 77 & 856 & 103 & 524 & 31 & 241 & 334 & 418 & 0 & 433 & 15 \\
\hline Ponto 1 & 675 & 117 & 25 & 62 & 420 & 302 & 11 & 70 & 416 & 0 & 0 & 0 \\
\hline Ponto Final 1 & 3653 & 857 & 3475 & 1010 & 3942 & 1103 & 3722 & 989 & 2847 & 632 & 2612 & 744 \\
\hline Testemunha 2 & 326 & 57 & 410 & 37 & 257 & 94 & 288 & 89 & 226 & 0 & 245 & 0 \\
\hline Ponto 2 & 1897 & 448 & 16 & 120 & 2526 & 541 & 28 & 70 & 2987 & 181 & 13 & 0 \\
\hline Ponto 3 & 2020 & 512 & 19 & 498 & 1912 & 131 & 11 & 120 & 2638 & 238 & 20 & 225 \\
\hline Ponto Final 2 & 735 & 346 & 412 & 317 & 820 & 280 & 569 & 88 & 527 & 0 & 341 & 16 \\
\hline TOTAL & 8980 & 2280 & 3947 & 2007 & 9620 & 2357 & 4341 & 1337 & 9415 & 1051 & 2986 & 985 \\
\hline
\end{tabular}

Observação: O total não inclui o ponto testemunha.

Tabela 4. Controle de imaturos de Simuliidae no ribeirão Cambé, Londrina-PR, pelo produto Teknar® utilizando-se da nova metodologia.

\begin{tabular}{|c|c|c|c|c|c|c|c|c|c|c|c|c|}
\hline \multirow{3}{*}{ Pontos de coleta } & \multicolumn{12}{|c|}{ Datas de Coleta } \\
\hline & \multicolumn{2}{|c|}{$03 / \mathrm{Abr}-0 \mathrm{hr}$} & \multicolumn{2}{|c|}{$04 / \mathrm{Abr}-24 \mathrm{hr}$} & \multicolumn{2}{|c|}{ 17/Abr - 0 hr } & \multicolumn{2}{|c|}{ 18/Abr - $24 \mathrm{hr}$} & \multicolumn{2}{|c|}{$24 / \mathrm{Abr}-0 \mathrm{hr}$} & \multicolumn{2}{|c|}{$25 / \mathrm{Abr}-24 \mathrm{hl}$} \\
\hline & Larva & Pupa & Larva & Pupa & Larva & Pupa & Larva & Pupa & Larva & Pupa & Larva & Pupa \\
\hline Testemunha 1 & 588 & 0 & 603 & 0 & 538 & 0 & 522 & 4 & 371 & 2 & 394 & 0 \\
\hline Ponto 1 & 541 & 3 & 0 & 0 & 308 & 0 & 0 & 0 & 413 & 0 & 0 & 0 \\
\hline Testemunha 2 & 2981 & 23 & 2812 & 0 & 933 & 3 & 1010 & 0 & 1123 & 4 & 1643 & 0 \\
\hline Ponto 2 & 428 & 2 & 4 & 0 & 261 & 12 & 0 & 0 & 284 & 0 & 0 & 0 \\
\hline Ponto 3 & 2314 & 35 & 19 & 11 & 1189 & 15 & 4 & 0 & 1048 & 15 & 16 & 2 \\
\hline Ponto 4 & 412 & 13 & 0 & 0 & 39 & 21 & 0 & 0 & 277 & 5 & 0 & 0 \\
\hline TOTAL & 3695 & 53 & 23 & 11 & 1797 & 48 & 4 & $\mathbf{0}$ & 2022 & 20 & 16 & 2 \\
\hline
\end{tabular}

Observação: O total não inclui o ponto testemunha. 
No ribeirão Tamapuã, também foi utilizada a metodologia tradicional (Figura 3A). Foi aplicado $170 \mathrm{~mL}$ para um trecho de $750 \mathrm{~m}$ de carreamento, obtendo-se uma mortalidade de 13,87\% (Tabela 5). Com a utilização da nova metodologia obteve-se uma mortalidade de $99,32 \%$ (Tabela 6).

Tabela 5. Controle de imaturos de Simuliidae no ribeirão Tamapuã, Arapongas - PR, pelo produto Teknar, pela metodologia tradicionalmente utilizada para cálculo de dosagem.

\begin{tabular}{|c|c|c|c|c|c|c|c|c|c|c|c|c|}
\hline \multirow{3}{*}{$\begin{array}{l}\text { Pontos de } \\
\text { Coleta }\end{array}$} & \multicolumn{12}{|c|}{ Datas de coleta } \\
\hline & \multicolumn{2}{|c|}{ 13/Out - 0 hr } & \multicolumn{2}{|c|}{ 14/Out $-24 \mathrm{hr}$} & \multicolumn{2}{|c|}{ 20/Out - 0 hr } & \multicolumn{2}{|c|}{ 21/Out - 24 hr } & \multicolumn{2}{|c|}{ 03/Nov - 0 hr } & \multicolumn{2}{|c|}{ 04/Nov - 24 hr } \\
\hline & Larva & Pupa & Larva & Pupa & Larva & Pupa & Larva & Pupa & Larva & Pupa & Larva & Pupa \\
\hline Testemunha & 781 & 114 & 855 & 90 & 874 & 43 & 779 & 285 & 997 & 76 & 936 & 19 \\
\hline Ponto 1 & 1896 & 113 & 11 & 225 & 2066 & 366 & 8 & 476 & 1993 & 741 & 7 & 666 \\
\hline Ponto 2 & 3258 & 956 & 3121 & 1232 & 3109 & 1118 & 3277 & 391 & 3275 & 632 & 3387 & 744 \\
\hline Ponto 3 & 2633 & 36 & 2523 & 836 & 2937 & 744 & 2716 & 357 & 3101 & 265 & 3222 & 312 \\
\hline Ponto 4 & 1896 & 126 & 1965 & 169 & 2523 & 639 & 2498 & 183 & 2723 & 395 & 2659 & 652 \\
\hline Ponto 5 & 2399 & 632 & 2684 & 657 & 3742 & 339 & 3888 & 116 & 3758 & 552 & 3611 & 681 \\
\hline TOTAL & 12082 & 1863 & 10304 & 3119 & 14377 & 3206 & 12387 & 1523 & 14850 & 2585 & 12886 & 3055 \\
\hline
\end{tabular}

Observação: O total não inclui o ponto testemunha.

Tabela 6. Controle de imaturos de Simuliidae no ribeirão Tamapuã, Arapongas - PR, pelo produto Teknar ${ }^{\circledR}$ utilizandose da nova metodologia.

\begin{tabular}{|c|c|c|c|c|c|c|c|c|c|c|c|c|}
\hline \multirow{3}{*}{$\begin{array}{l}\text { Pontos de } \\
\text { Coleta }\end{array}$} & \multicolumn{12}{|c|}{ Datas de Coleta } \\
\hline & \multicolumn{2}{|c|}{ 10/Nov - 0 hr } & \multicolumn{2}{|c|}{ 11/Nov - $24 \mathrm{hr}$} & \multicolumn{2}{|c|}{ 17/Nov - 0 hr } & \multicolumn{2}{|c|}{ 18/Nov - 24 hr } & \multicolumn{2}{|c|}{ 24/Nov - 0 hr } & \multicolumn{2}{|c|}{$25 /$ Nov $-24 \mathrm{hr}$} \\
\hline & Larva & Pupa & Larva & Pupa & Larva & Pupa & Larva & Pupa & Larva & Pupa & Larva & Pupa \\
\hline Testemunha & 882 & 119 & 971 & 94 & 973 & 112 & 927 & 288 & 1392 & 175 & 1005 & 161 \\
\hline Ponto 1 & 2741 & 227 & 11 & 211 & 2074 & 378 & 17 & 628 & 3776 & 147 & 12 & 98 \\
\hline Ponto 2 & 4963 & 915 & 16 & 920 & 3726 & 1085 & 10 & 476 & 3296 & 236 & 8 & 268 \\
\hline Ponto 3 & 3985 & 360 & 21 & 342 & 3993 & 752 & 27 & 244 & 3568 & 562 & 18 & 426 \\
\hline Ponto 4 & 4375 & 285 & 20 & 216 & 4190 & 666 & 30 & 195 & 3722 & 409 & 16 & 335 \\
\hline Ponto 5 & 3175 & 658 & 13 & 657 & 3265 & 319 & 31 & 168 & 3785 & 724 & 10 & 744 \\
\hline TOTAL & 19239 & 2445 & 81 & 2346 & 17248 & 3200 & 115 & 1711 & 18147 & 2078 & 64 & 1871 \\
\hline
\end{tabular}

Observação: O total não inclui o ponto testemunha.

Analisando os resultados obtidos, os ribeirões Cambé e o Tamapuã, que apresentavam variações geomorfológicas capazes de influenciar na velocidade e quantidade de transporte de partículas pela água, foi possível verificar que a metodologia tradicional não foi eficiente no controle dos imaturos de simulídeos em toda a distância proposta. Nestas condições, produtos a serem aplicados devem sofrer o efeito de decantação depositando-se no fundo do leito, dificultando o carreamento do mesmo.
A nova proposta de metodologia mostrou-se eficiente, controlando em média 99,43\% das larvas de simulídeos no ribeirão Cambé com a redução de $40,49 \%$ da quantidade de produto aplicado. Esta redução foi possível pelo fato das condições geomorfológicas do ribeirão terem sido levadas em consideração, uma vez que apresenta cerca de $50 \%$ de sua extensão com área alagada, não sendo necessário o controle. Para o ribeirão Tamapuã houve aumento de $61,71 \%$ na quantidade de produto 
a ser aplicado, devido a presença de piscinas naturais. Portanto o consumo de produto, quando utilizado a nova metodologia, pode aumentar ou diminuir dependendo das características de cada ribeirão, pois se apresentar um alto número de fragmentações de doses, o consumo pode aumentar significativamente.

A mortalidade obtida com esta última metodologia foi semelhante a encontrada por Petry et al. (2004), em riacho localizado na cidade de Almirante Tamandaré no estado do Paraná, onde conseguiram, utilizando controle integrado, uma redução de até 99,97\% das larvas de simulídeos. Ruas-Neto (1984), trabalho pioneiro de controle de simulídeos a campo, obteve uma mortalidade inferior a $90 \%$ quando a vazão do ribeirão foi superior a $5 \mathrm{~m}^{3}$. $\min ^{-1}$, e mortalidades irregulares quando a vazão foi inferior a $5 \mathrm{~m}^{3} \cdot \mathrm{min}^{-1}$. Estes resultados foram inferiores aos obtidos por esta pesquisa, pois as vazões nos três ribeirões estudados foram superiores a $15 \mathrm{~m}^{3}$. $\mathrm{min}^{-1}$ porém as mortalidade obtidas foram maiores que os $90 \%$ do que as encontrados por Ruas-Neto et al. (1985).

O controle verificado para as diferentes espécies que colonizavam os ribeirões, coincide com o encontrado por Petry (2005), onde em um estudo de suscetibilidade das espécies de simulídeos a diferentes formulados a base de B.t.i., verificou que as espécies Simulium perflavum Roubaud, 1906, Simulium (Inaequalium) inaequale Peterson e Shannon, 1927, S. pertinax, Simulium (Inaequalium) subnigrum Lutz, 1910 e Simulium (Thyrsopelpa) orbitale Lutz, 1910 responderam de forma semelhante quanto a mortalidade aos produtos. Resultados divergentes, como os de Lacey e Lacey (1981), que ao investigarem a suscetiblidade de seis espécies de Simulium, coletadas no campo, concluíram que, dentre outras, Simulium vittatum Zetterstedt, 1838 e Simulium argus Williston, 1893, foram consideravelmente mais sensíveis aos efeitos do B.t.i.

As eficiências obtidas na utilização das duas metodologias em estudo apresentaram diferenças significativas através do teste de Tukey a nível de $5 \%$ de significância (Tabela 7). O ponto Testemunha (ponto isento de ação do larvicida) não foi utilizado para as análises estatísticas e as diferenças entre o coletado em dias diferentes foi, em média inferior a 5\%, o que pode ser considerado variação natural influenciada pelo próprio comportamento do animal.

Tabela 7. Controle de larvas de simulídeos obtido pelo produto Teknar ${ }^{\circledR}$, comparando-se duas metodologias: Metodologia Tradicional e Nova Metodologia com a subdivisão de dose.

\begin{tabular}{|c|c|c|c|c|c|c|c|c|c|c|c|c|}
\hline \multirow[t]{4}{*}{ Metodologia } & \multicolumn{12}{|c|}{ Ribeirões Estudados } \\
\hline & \multicolumn{4}{|c|}{ Cambé } & \multicolumn{4}{|c|}{ Tamapuã } & \multicolumn{4}{|c|}{ Cafezal } \\
\hline & \multicolumn{2}{|c|}{ Pontos } & \multicolumn{2}{|c|}{ Testemunha } & \multicolumn{2}{|c|}{ Pontos } & \multicolumn{2}{|c|}{ Testemunha } & \multicolumn{2}{|c|}{ Pontos } & \multicolumn{2}{|c|}{ Testemunha } \\
\hline & Aplicação & $24 \mathrm{hr}$ após & $0 \mathrm{hr}$ & $24 \mathrm{hr}$ & Aplicação & $24 \mathrm{hr}$ após & $0 \mathrm{hr}$ & $24 \mathrm{hr}$ & Aplicação & $24 \mathrm{hr}$ após & $0 \mathrm{hr}$ & $24 \mathrm{hr}$ \\
\hline Tradicional & $28015 \mathrm{~A} \mathrm{a}$ & $11274 \mathrm{Bb}$ & $2354 \mathrm{Cc}$ & $2473 \mathrm{Cc}$ & 41309Dd & 35577Df & $2652 \mathrm{Ll}$ & $2570 \mathrm{Ll}$ & $11226 \mathrm{M}$ & $98 \mathrm{~N}$ & $2682 \mathrm{P}$ & $2580 \mathrm{P}$ \\
\hline Nova & $7514 \mathrm{Bb}$ & $43 \mathrm{Aa}$ & $6534 \mathrm{Cc}$ & $6984 \mathrm{Cc}$ & $38334 \mathrm{Gd}$ & $260 \mathrm{Ij}$ & $3247 \mathrm{Ll}$ & $2903 \mathrm{Ll}$ & * & $*$ & & \\
\hline CV \% & \multicolumn{4}{|c|}{5.11} & \multicolumn{4}{|c|}{1.02} & \multicolumn{4}{|c|}{1.89} \\
\hline DMS & \multicolumn{4}{|c|}{1.2115429} & \multicolumn{4}{|c|}{0.1598702} & \multicolumn{4}{|c|}{0} \\
\hline
\end{tabular}

Número de larvas seguidos pela mesma letra maiúscula na linha e minúscula na coluna não difere entre si pelo teste de Tukey no nível de 5\% de significância. 
Os fitilhos utilizados foi na cor verde escuro, pois em testes preliminares verificou-se uma maior postura de ovos nesta cor, resultados que diferem de Petry (2005), onde verificou uma preferência por fitilhos de cores claras, tais como amarelos e brancos. A divergência entre os resultados obtidos pelo autor acima citado, pode estar relacionado ao comportamento de diferentes espécies de cada região de estudo.

Barragens feitas nos pequenos ribeirões, principalmente para represamento visando a retirada de água para irrigação ou para abastecimento diverso, pode bloquear totalmente o carreamento. As piscinas naturais podem ser formadas por queda de água com alturas consideráveis. Esta situação leva a formação de "remansos" com maior profundidade e provoca uma circulação da água no sentido horizontal e vertical, com refluxos pelas laterais e a saída é feita pela borda superior da "piscina". Outras são quedas menores com formação de piscinas mais rasas e com fluxo mais rápido da água. Existem ainda a formação de remansos naturais caracterizando-se por uma área mais profunda, normalmente sem pedras no fundo, com águas lênticas que se segue logo após a um trecho de corredeira. Todas essas situações devem ser levadas em consideração para a determinação do carreamento de produto bioinseticida, utilizado no controle de borrachudos. Desta forma uma vez detectada estas influências, trabalhos minuciosos de mapeamento do ribeirão devem ser realizados.

Em relação ao tempo necessário para aplicação, no ribeirão Cambé foi de $20 \mathrm{~min}$. para a metodologia tradicional, já para a nova proposta, com o aumento de um ponto de aplicação, houve um acréscimo de 10 min. Não foi considerado o tempo de deslocamento entre um ponto de aplicação e outro, já que a extensão não tratada estava entre o ponto inicial e o ponto final, não alterando portanto o tempo de deslocamento. No Ribeirão Tamapuã, pela metodologia tradicional foi gasto $10 \mathrm{~min}$. na aplicação, considerando que havia apenas um ponto de aplicação do B.t.i. e o produto seria carreado por toda a extensão estudada. Pela nova metodologia, quatro novos locais de tratamento foram acrescentados. Desta forma o tempo de aplicação aumentou para $50 \mathrm{~min}$, acrescido de 40 min. para o deslocamento, o que totaliza $90 \mathrm{~min}$.

Conclui-se que a nova proposta de metodologia de aplicação de bioinseticida é uma alternativa importante para o controle de simulídeos em ribeirões com características topográficas acentuadas. A metodologia tradicional não é recomendada para ribeirões semelhantes aos citados anteriormente, sendo indicada somente para o controle em ribeirões com leito regular.

Mais estudos são necessários para minimizar a mão-de-obra na aplicação da nova proposta de metodologia e para estabelecer qual é a amplitude da influência no carreamento em razão do tamanho, profundidade e característica da "piscina natural".

\section{Agradecimentos}

Ao programa de pós graduação em Agronomia da Universidade Estadual de Londrina. Ao $\mathrm{CNPq}$ pela concessão de bolsa de estudo. Ao pesquisador Dr. Milton Noberto Strieder pelo auxílio na identificação das espécies e ao acadêmico em Ciências Biológicas Murillo Bernardi, pela ajuda nas atividades de campo.

\section{Referencias}

AOKI, V.; HANS-FILHO, G.; RIVITTI, E. A.; DIAZ, L. A.; ITO, L. M. Perfil histórico da imunopatogenia do pênfigo foliáceo endêmico (fogo selvagem). Anais Brasileiros de Dermatologia, Rio de Janeiro, v. 88, n. 3, p. 287-292, 2005.

BECKER, N.; ZGOMBA, M.; LUDWIG, M.; PETRIC, D.; RETTICH, F. Factors influencing the activity of Bacillus thuringiensis var. israelensis treatments. Journal of the American Mosquito Control Association, Panama, v. 8, n. 3, p. 285-289, 1992. 
BROWN, M. D.; DARRAN, T.; PAUL, M.; JACK, G. G.; BRIAN, H. K. Laboratory and field evalution of the efficacy of four insecticides for Aedes vigilax (Diptera: Culicidae) and toxicity to the nontarget shrimp Leander tenuicornis (Decapoda: Palaemonidae). Journal of Economic Entomology, Aimes, v. 92, n. 5, p. 1045-1051, 1999.

BROWN, M. D.; DARRAN, T.; TONYA, M. W.; BRIAN, H. K. Laboratory and field evalution of the efficacy of Vectobac $12 \mathrm{AS}$ against Culex sitiens (Diptera: Culicidae) larvae. Journal of the American Control Association, Panama, v. 14, n. 2, p. 183-185, 1998.

BROWN, M. D.; TONYA, M. W.; SUSANNAH, G.; JACK, G. G.; DAVID, P. E.; BRIAN, H. K. Toxicity of insecticides for control of freshwater Culex annulirostris (Diptera: Culicidae) to the nontarget shrimp, Caradina indistincta (Decapoda: Atyidae). Journal of Economic Entomology, Aimes, v. 93, n. 3, p. 667-671, 2000.

CAVADOS, C. F. G.; FONSCECA, R. N.; CHAVES, J. Q.; ARAÚJO-COUTINHO, C. J. P. C.; RABINOVITCH, L. A new black fly isolate of Bacillus thuringiensis autoagglutinating strain highly toxic to Simuliium pertinax (Kollar) (Diptera, Simuliidae) larvae. Memórias Instituto Oswaldo Cruz, Rio de Janeiro, v. 100, n. 7, p. 795-797, 2005.

CAVADOS, C. F. G.; FONSCECA, R. N.; CHAVES, J. Q.; RABINOVITCH, L.; ARAÚJO-COUTINHO, C. J. P. C. Identification of entomopathogenic Bacillus isolated from Simulium (Diptera: Simuliidae) larvae and adults. Memórias Instituto Oswaldo Cruz, Rio de Janeiro, v. 96, n. 7, p. 1017-1021, 2001.

CHUNG, Y. K.; PHUA-LAM, S.G.; CHUA, Y. T.; YATIMAN, R. Evaluation of biological and chemical inseticide mixture against Aedes aegypti larvae and adults by thermal fogging in Singapore. Medical and Veterinary Entomology, Canadá, v. 15, p. 321-327, 2001.

COSCARÓN, S.; WYGODZINSKY, P. Notas sobre Simulidos neotropicales. VII. Sobre los subgeneros Psaroniocompsa Enderlein y Inaequalim subgen. nov. Arquivos de Zoologia, São Paulo, v. 31, n. 2, p. 37-103, 1984.

COSCARÓN, S. Fauna de agua dulce de la República Argentina. Fascículo 2. Buenos Aires: Fundación para la Educación, la Ciencia y la Cultura. 1991. 297 p.

CUNHA, M. C. I. Simulídeos (borrachudos). In: MARCONDES, C. B. Entomologia médica e veterinária. São Paulo. Atheneu, 2001, p. 31-47.

FILLINGER, U.; KNOLS, B. G. J.; BECKER, N. Efficacy and efficiency of new Bacillus thuringiensis var. israelensis and Bacillus sphaericus formulations against
Afrotropical anophelines in Western Kenya. Tropical Medicine and International Health, University Logo, v. 8, n. 1, p. 37-47, 2003.

HAMADA, N.; ALE-ROCHA, R.; LUZ, S. L. B. Description of Simulium damascenoi (Diptera: Simuliidae) male and the blackfly species from the state of Amapá, Brazil. Memórias do Instituto Oswaldo Cruz, Rio de Janeiro, v. 98, n. 3, p. 353-360, 2003.

LACEY, L. A.; UNDEEN, A. H. The effect of formulation, concentration, and application time on the efficacy of Bacillus thuringiensis (H14) against black fly larvae under natural conditions. Journal of Economic Entomology, Aimes, v. 77, p. 412-418, 1984.

LACEY, L. A. E.; LACEY, J. M. The larvicidal activity of Bacillus thuringiensis var. israelensis $(\mathrm{H}-4)$ against mosquitoes os the Central Amazon Basin. Mosquito New, New York, v. 41, n. 2, p. 266-270, 1981.

LOZOVEI, A. L.; PETRY, F.; SANTOS NETO, L. G. Survey of the Simulium species (Diptera, Simuliidae), Riacho dos Padres, almirante Tamandaré munipality, Paraná, Brazil. Revista Brasileira de Entomologia, Curitiba, v. 48, n. 1, p. 91-94, 2004.

NASCIMENTO, E. S.; FIGUEIRÓ, R.; BECNEL, J. J.; ARAÚJO-COUTINHO, C. J. P. C. Influence of temperature on mirosporidia infections in a natural population of Simulium pertinax Kollar, 1832 (Diptera: Simuliidae). Brazilian Journal of Biology, São Carlos, v. 67, n. 3, p. 519-526, 2007.

NAYAR, J. K.; KNIGHT, J. W.; ALI, A.; CARLSON, D. B. E.; O'BRYAN, P. D. Laboratory evalution of biotic and abiotic factors tha may influence larvicidal activity of Bacillus thuringiensis serovar israelensis against two Florida mosquito species. Journal of the American Mosquito Control Association, Panama, v. 15, n. 1, p. 3242, 1999.

PETRY, F. Aspectos biológicos de fertilidade e ciclo evolutivo de espécies de Simulium (Diptera, Simuliidae) e susceptibilidade de suas larvas em bioensaios laboratoriais com formulados de Bacillus thuringiensis israelensis em aparato elaborado. 2005. Tese (Mestrado em Ciencias Biológicas) - Departamento de Ciências Biológicas. Universidade Federal do Paraná, Curitiba.

PETRY, F.; LOZOVEI, A. L.; EHLERS, S. C. Eggs Fertility and Biological Aspects of the Life Cycle in Natural populations of Simulium (Diptera, Simuliidae). Brazilian Archives of Biology and Technology, Brazil, v. 49, n. 5, p. 799-805, 2006.

PETRY, F.; LOZOVEI, A. L.; FERRAZ, M. E.; SANTOS NETO, L.G. Controle integrado de espécies de Simulium (Diptera, Simuliidae) por Bacillus thuringiensis e 
manejos mecânicos no riacho e nos vertedouros de tanque de piscicultura, Almirante Tamandaré, Paraná, Brasil. Revista Brasileira de Entomologia, Curitiba, v. 48, n. 1, p. 127-132, 2004.

POLLOM, T. E. V. Desenvolvimento de bioprocessadores para produção de biomassa de delta-endotoxina com cepa BRO1 de Bacillus thuringiensis israelensis Berliner, 1915, visando o controle biológico de Aedes aegypti Linnaeus, 1762 (Diptera: Culicidae). 2003. Tese (Doutorado em Ciências Biológicas) - Departamento Ciências Biológicas. Universidade Federal do Paraná, Curitiba.

RIO GRANDE DO SUL (Estado). Secretaria Estadual da Saúde. Centro Estadual de Vigilância em Saúde. Simulídeos: Programa Estadual Rio Grande do Sul, Brasil. Guia para orientação aos municípios sobre o manejo integrado, controle e gestão de insetos da Família Simuliidae (Díptera: Nematocera). Porto Alegre: CEVS, 2006. $41 \mathrm{p}$.

RODRIGUES, I. B.; WANDERLI, P. T.; JOSÉ, M. C. S. D. Studies on the Bacillus sphaericus larvicidal activity against malarial vector species in Amazonia. Memosrias do Instituto Oswaldo Cruz, Rio de Janeiro, v. 93, n. 4, p. 441-444, 1998.

RUAS NETO, A. L. Bacillus thuringiensis var. israelensis como alternativa no controle de simulídeos no Rio Grande do Sul. 1- Susceptibilidade a campo. Boletim de Saúde. Porto Alegre, v. 11, n. 2, p. 21-26, 1984.
RUAS NETO, A. L.; SOUZA, M. A. T.; SEVERINO, S.; MELO, J. L. B.; SILVEIRA, S. M.; DE FORTES, N. D. F. Controle integrado de Simuilium (Chirostilbia) pertinax Kollar, 1832. 1. Utilização de Bacillus thuringiensis var. israelensis em três municípios do Rio Grande do sul. Boletim de Saúde, Porto Alegre, v. 12, p. 17-20, 1985.

STRIEDER, M. N. Controle eficiente dos borrachudos. Ciência Hoje, Rio de Janeiro, v. 36, p. 70-71, 2005.

Espécies de Simulídeos (Diptera, Nematocera, Simuliidae) no Rio Grande do Sul, Brasil: Distribuição Geográfica. Entomologia y Vectores, Rio de Janeiro, v. 11, n. 1, p. 113-143, 2004.

STRIEDER, M. N.; CORSEUIL, E.; PY-DANYEL, V. Espécies do gênero Simulium (Diptera: Simuliidae) ocorrentes no Rio Grande do Sul-Brasil, com chaves para sua identificação. Acta Biológica Leopoldensia, São Leopoldo, v. 14, n. 2, p. 53-74, 1992.

STRIEDER, M. N.; SANTOS JUNIOR, J. E.; PES, A. M. O. Diversidade e distribuição de Simuliidae (Diptera, Nematocera) no gradiente longitudinal da bacia do rio dos Sinos, no Rio Grande do Sul, Brasil. Entomologia y Vectores, Rio de Janeiro, v. 9, n. 4, p. 527-540, 2002.

STRIEDER, M. N.; SANTOS, J. E. dos; VIEIRA, E. M. Distribuição, abundância e diversidade de Simuliidae (Diptera) em uma bacia hidrográfica impactada no sul do Brasil. Revista Brasileira de Entomologia, Curitiba, v. 50, n. 1, p. 119-124, 2006. 